\title{
Development of a mobile-based self-management health alarm program for obese children in South Korea and a test of its feasibility for metabolic outcomes: A study based on the information-motivation-behavioral skills model
}

\author{
Jihea Choi ${ }^{1}$, Yon Chul Park ${ }^{2}$, Sarah Choi ${ }^{3}$ \\ ${ }^{1}$ Assistant Professor, Department of Nursing, Yonsei University Wonju College of Medicine, Wonju; ${ }^{2}$ Assistant Professor, Department of Family Medicine, Wonju \\ Severance Christian Hospital, Wonju, Korea; ${ }^{3}$ Associate Professor, School of Nursing, University of California in Los Angeles, CA, USA
}

Purpose: This study aimed to develop a mobile-based self-management health alarm (MSHA) program for modifying obese children's lifestyle based on the information-motivation-behavioral skills (IMB) model and to test its feasibility. Methods: A methodological study for the development of the MSHA program and pilot study with a one-group pretest-posttest design for feasibility testing was conducted. The MSHA program was designed to provide obesity-related information (I), monitor daily diet and exercise, provide motivational text messages (M), and enhance healthy diet and exercise skills (B) via a mobile-based web platform. In the feasibility test, six obese children participated in the 4-week program, and the number of days per week that they achieved their goals and differences in metabolic components were assessed. Data were analyzed using descriptive statistics and the Wilcoxon signed-rank test. Results: Participants successfully achieved their diet and exercise goals $\geq 5$ days per week. Body mass index $(\mathrm{z}=-1.99, p=.046)$, waist circumference $(\mathrm{z}=-2.20, p=.028)$, and triglyceride levels $(\mathrm{z}=-2.21, p=.027)$ significantly decreased. Conclusion: The MSHA program showed positive effects on health behaviors and metabolic syndrome risk. The program may be effective in improving metabolic syndrome in obese children by promoting self-health management behaviors.

Key words: Child; Obesity; Health behavior; Self-management

\section{Corresponding author Jihea Choi \\ https://orcid.org/0000-0003-1881-6342}

Department of Nursing, Yonsei University Wonju College of Medicine, 20 Ilsan-ro, Wonju 26426, Korea

TEL +82-33-741-0379 FAX +82-33-743-9490

E-MAIL jiheachoi@yonsei.ac.kr

*This study was supported by a National Research Foundation of Korea (NRF) grant funded by the Korean government (No. NRF-2017R1C1B5076837).

Received Oct 13, 2020 Revised Nov 16, 2020 Accepted Nov 17, 2020

\section{INTRODUCTION}

Childhood obesity is one of the most serious global public health challenges of the 21st century, and its prevalence is increasing [1]. The number of children with obesity worldwide has increased more than tenfold, from 11 million to 124 million in just 40 years [1]. In Korea, where this study was conducted, the prevalence rate of obesity in children and adolescents increased from $11.2 \%$ in 2008 to $17.3 \%$ in 2017 [2]. This steady increase in prevalence requires an active and effective intervention plan to decrease obesity in children.

Childhood obesity is a health concern because it leads to adulthood obesity and increases the risks of metabolic syndrome, in which factors such as abdominal obesity, hypertension, dyslipidemia, and glucose intolerance are compounded [3]. Previously, metabolic syndrome was considered a health problem confined only to adults. However, a study conducted on obese children in 195 countries from 1980 to 2015 showed that early diagnosis and prevention through screening for metabolic risk factors in childhood helped reduce morbidity and mortality of type 2 diabetes mellitus and atherosclerotic cardiovascular disease at a younger age in adults [4]. This previous study suggested that early interventions for (a) This is an Open Access article distributed under the terms of the Creative Commons Attribution NonCommercial License (http://creativecommons.org/licenses/by-nc/4.0/) which permits unrestricted noncommercial use, distribution, and reproduction in any medium, provided the original work is properly cited. 
childhood obesity will play an extremely important role in the prevention of metabolic syndrome in adulthood. Hence, evaluating the impact of interventional programs on the management of metabolic components in cases of childhood obesity will be meaningful for efforts to decrease the prevalence of type 2 diabetes mellitus and atherosclerotic cardiovascular disease in adults.

Studies on therapeutic approaches to childhood obesity management have suggested that reducing the intake of calories and increasing exercise are the best steps for the prevention and primary treatment of obesity [5]. Moreover, it was found that persistent self-management to maintain healthy behaviors was the most important factor for preventing obesity [5]. Considering that obesity in childhood is closely related to obesity-related complications in adulthood, such as type 2 diabetes mellitus and atherosclerotic cardiovascular disease, promoting self-management among obese children is the most important task of the current era and in the future [3]. While many interventions for childhood obesity management have been developed and their effectiveness has been demonstrated, it has been found that the most important factor in lifestyle management interventions to reduce obesity is that interventions be theory-based [6].

Various theories have been proposed to explain human health behavior. In particular, the information-motivationbehavioral skills (IMB) model, which was developed as a theoretical basis for promoting health behavior, has been shown to be a useful theoretical framework and has been widely used to explain complex health behaviors such as human immunodeficiency virus prevention, breast self-examination, and diabetes self-care in adults $[7,8]$, as well as obesity management, smoking cessation, and diabetes self-care in childhood and adolescence [9-11]. The IMB model presents a clear causal relationship between behavior-related factors, including information (knowledge necessary for behavior change), motivation (individual beliefs or attitudes that induce behavior change), and behavior skills (confidence in performing the changed behavior) [8]. It has been proven to be effective in helping children with chronic diseases to modify their lifestyle and sustain healthy behaviors $[9,10]$. However, only one study conducted in the USA has used the IMB model to develop an intervention program for self-management of obese children, who require persistent diet management and exercise [11]. It is therefore meaningful to develop and evaluate a self-management program for childhood obesity management based on the IMB model, which has proven its theoretical strength in persistent health behavior among children.

In particular, late school-age is a period in which school health education begins to promote health behavior through the acquisition of health-related knowledge, values, and skills
[12]. Effective interventions based on the IMB model would provide the information, motivation, and behavioral skills for obesity management needed to change self-health behaviors in an easy and simple manner for children. However, many interventional programs for childhood obesity management have focused on providing a large amount of information on obesity-related health issues to children or on raising children's awareness of their health status [6,13]. These interventional programs have usually consisted of one-way interactions, from health care providers to children, limiting children's active participation and motivation to change [6]. Therefore, if obese children directly report their diet and exercise behavior to health care providers, and health care providers provide the necessary information and motivation according to the children's reports, it will be easier for children to form healthy diet and exercise behavior through a two-way interaction between children and health care providers.

Rapid technological advancements have led to innovative changes in the provision of healthcare services and information. Mobile-based healthcare interventions have been actively developed worldwide since 2007, when the iPhone, a breakthrough smartphone technology, was released [14]. Newly developed mobile-based intervention programs for children have been innovative in that they not only easily provide information of interest to the user at a tremendous speed, but also allow health care providers and patients to easily interact with each other [14]. Additionally, the development of wearable devices that measure the number of steps a person takes and mobile-phone apps has expanded the possibilities for users' self-monitoring and self-management of their lifestyle anytime, anywhere, and at a low cost $[14,15]$. Moreover, recent statistics showed that more than $91.4 \%$ of children and adolescents use a smartphone in Korea, which has led them to being called the "mobile native generation"[16]. This suggests that mobile-based intervention programs have great potential for positively influencing self-health management practices for childhood obesity.

Therefore, the authors intended to develop and test the feasibility of a mobile-based self-management health alarm (MSHA) program based on the IMB model in a simple and easy manner that would be suitable for late school-age children to monitor and manage their health behaviors using mobile devices. The MSHA program focused on enhancing obesity self-management knowledge, motivational attitudes, and health behavioral skills in children by having them self-monitor and manage their diet and exercise using mobile-based educational materials and supportive mobile text messages. We hypothesized that the MSHA program would serve as an effective self-management tool for children to monitor and manage childhood obesity. 


\section{Purpose}

The purpose of this study was to develop and pilot-test the feasibility and effectiveness of the MSHA program based on the IMB model. The MSHA program was developed as a web platform aimed at giving children the ability to perform daily self-monitoring and management of their diet and exercise behavior to provide health behavior information, motivation, and skills. The authors also evaluated the program's final effectiveness in changing and maintaining children's health behavior and improving their metabolic components as result of persistent health behavior.

\section{METHODS}

This study consisted of two phases. Phase I was a methodological study focused on the development of the MSHA program based on the IMB model using a wearable pedometer and a mobile-phone diet application (app) for obese children. Phase II was a pilot study using a one-group pretest-posttest design to evaluate the feasibility and effectiveness of the MSHA program.

\section{Phase I: Development of the Mobile-based Self-man- agement Health Alarm Program}

\section{1) Information-motivation-behavioral skills model as a theoret- ical framework}

The MSHA program was based on the IMB model, The IMB model provides a clear causal relationship between factors promoting health behaviors in patients with chronic diseases and successful self-management, which plays an important role in behavior change. The IMB model provides the information necessary for changing health behaviors through successful self-management and induces motivation for the establishment of behavioral skills, resulting in behavior change. It is suitable for promoting health behaviors of patients with chronic diseases [8]. The conceptual framework of the MSHA program developed for obese children is shown in Figure 1.

\section{2) Mobile-based self-management health alarm program devel-} opment process

To develop the MSHA program, the authors (1) reviewed and appraised the existing interventional programs for the management of obesity in children, (2) constructed the specific strategies of the program based on the IMB model, (3) evaluated the content validity of the developed program using a group of experts (three nursing professors and one medical professor), and (4) revised the program based on the experts' recommendations and implemented it as a mobile-based program.

\section{Phase II: Feasibility Test}

\section{1) Participants and recruitment}

The number of participants required in this pilot study was calculated using the effect size of a similar previous study that aimed to assess the effectiveness of an obesity management program developed for school-age children [17]. Using the G*Power 3.1.9.2 program, the Wilcoxon signed-rank test for one sample was selected as our statistical method, and an effect size of 1.38 , significance level of .05 , and power of .80 were input as parameters. Finally, a sample size of six participants was calculated.

The authors individually contacted school nurses who wished to participate in this study based on the explanation of the research presented to the Elementary School Nurse Association in W City in Korea. Three school nurses who understood the purpose of the study and assisted with participants' recruitment responded that they would like to join. The se-

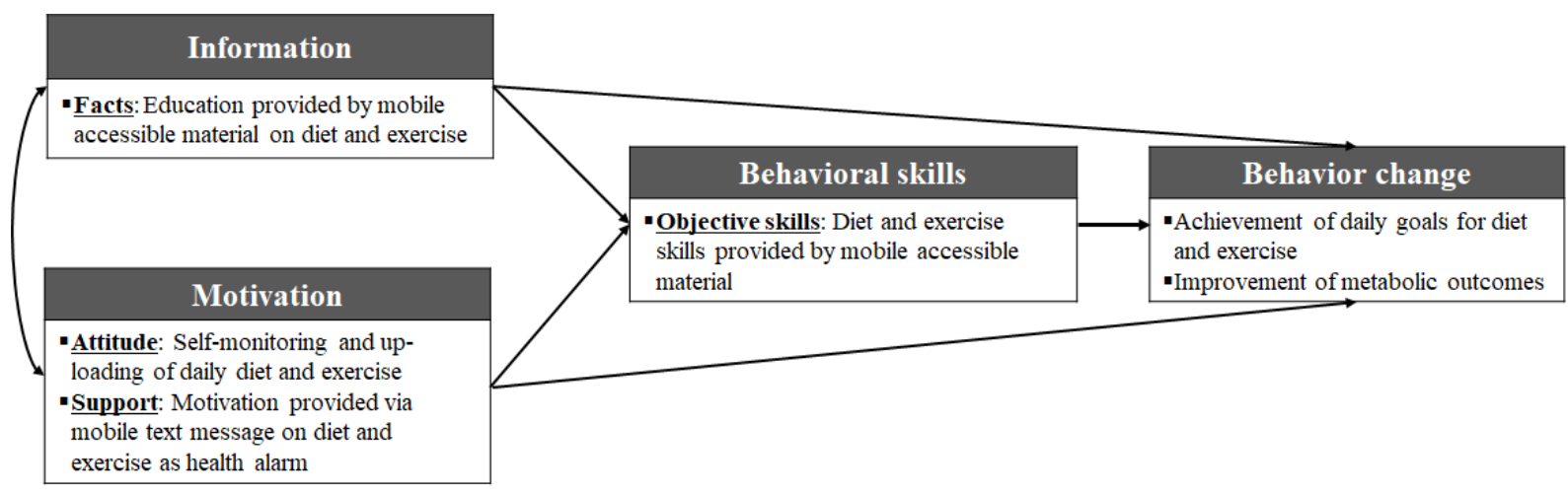

Figure 1. Conceptual framework of the mobile-based self-management health alarm (MSHA) program based on the informationmotivation-behavioral skills (IMB) model. 
lection criteria for participants in this study were children aged $\geq 10$ years with a body mass index (BMI) $\geq 95$ th percentile as defined by the 2017 Korean National Growth Charts [18] who could use mobile devices, wearable pedometers, and diet phone apps by themselves. Using the results of a school health examination, the school nurses recruited suitable participants. The authors then contacted the legal guardian of each study participant and explained the purpose and process of the study. Six students (two each aged 10, 11, and 12 years) were recruited and enrolled in the study with their legal guardian's permission for study participation. Children who met the following conditions were excluded from the study: current medical treatments related to obesity, diabetes, metabolic syndrome, and others; a medical history of congenital heart disease, diabetes, or hyperthyroidism; or a request by the participant or legal guardian to discontinue study participation during the study period. All study participants continued participation until the end of the study.

\section{2) Data collection and measurements}

Maintenance of diet and exercise health behavior, as well as levels of metabolic components, were evaluated to determine the effectiveness of the MSHA program. All data were collected from June 11, 2019 to July 19, 2019.

Based on the finding of a systematic review of online obesity prevention programs that interventions in childhood required at least 4 weeks to show their effectiveness [13], daily intake of calories and number of daily steps were collected via the MSHA platform through the daily uploading of information by participants for 4 weeks. Participants' daily intake of calories was calculated using a free public-use diet-diary phone app (DASHIN), in which the type and amount of consumed food are selected and the calories consumed are automatically calculated. Daily steps were calculated based on the number of steps measured by a wearable pedometer (Xiaomi Mi band 3, Xiaomi Corporation, Beijing, China), which participants were required to wear during all waking hours. Maintenance of health behavior was evaluated by the mean value of the number of days per week that diet and exercise goals were achieved.

The following variables were analyzed as metabolic components: BMI, waist circumference (WC), systolic blood pressure (SBP), diastolic blood pressure (DBP), triglyceride (TG) level, high-density lipoprotein-cholesterol (HDL-C) level, and fasting plasma glucose (FPG) level. Metabolic outcome data were collected twice: immediately before and immediately after the 4-week MSHA program. After all of the anthropometric variables (BMI, WC, SBP, DBP) were measured twice by two trained research assistants, the mean value of the double-measured data was used. BMI was calculated as weight divided by height squared $\left(\mathrm{kg} / \mathrm{m}^{2}\right)$. For BMI calculations, body weight was measured to the closest $0.1 \mathrm{~kg}$ on a medical balance scale, and height was measured to the closest $0.1 \mathrm{~cm}$ with a portable stadiometer. WC was measured at the midpoint between the lowest margin of the rib and uppermost lateral border of the iliac crest during expiration, using a flexible tape measure during minimal respiration. SBP and DBP were measured on the right arm after the subject had rested for 5 minutes in a seated position, using a mercury sphygmomanometer. Biochemical variables (TG, HDL-C, and FPG levels) were analyzed from a blood specimen that was collected by trained medical personnel after overnight fasting at the laboratory at the study director's affiliated university hospital. The specimens were analyzed within 1 hour after blood collection.

The general characteristics of the study participants (age, gender, sitting hours per day, numbers of days that participants exercised for over 60 minutes per week, and perceived health status) were surveyed before study participation. Parental medical history of diabetes, hypertension, and hyperlipidemia was surveyed through a simple parental medical history questionnaire that was provided to parents with the study consent form.

\section{3) Data analysis}

The collected data were analyzed using SPSS for Windows version 23.0 (IBM Corp., Armonk, NY, USA). The general characteristics of the subjects were analyzed using descriptive statistics. The maintenance of participants' health behavior was analyzed using the mean and standard deviation of the number of days per week that the goals were achieved. Improvement of metabolic components was analyzed using the Wilcoxon signed-rank test to compare the differences in metabolic components (BMI, WC, SBP, DBP, TG, HDL-C, and FPG) between the pretest and posttest results.

\section{4) Ethical considerations}

This study was approved by the Research Ethics Committee of the study director's affiliated university (Institutional Review Board number: CR319025). After potential participants were provided information about the purpose of the study, guarantees of anonymity and credibility, assurances that their participation was voluntary, and an explanation of the full process of the intervention study, written informed consent was obtained from the children and their legal guardians for participation in the study. As a reward for participation in this study, the wearable watch (Xiaomi Mi band 3, Xiaomi Corporation, Beijing, China) used in the study was provided to study participants at the end of the study. 


\section{RESULTS}

\section{Phase I: Development of a Mobile-based Self-man- agement Health Alarm Program}

\section{1) Step 1}

Review and appraisal of existing obesity management interventions A search for literature on existing obesity management interventions for children was conducted using the terms "child", "children", "obesity management", "mobile intervention", and "mobile health". The previous and existing intervention programs that were found included the weightmanagement elements of diet and exercise, nutritional education and counseling, behavioral treatments, psychological interventions, information sharing via social network services (SNS), and individual weight management counseling as major components $[6,14,19]$. These mobile-based interventions were effective in providing mediation anytime, anywhere, and at a lower cost than on-site interventions. However, although school-age children have the capacity to manage daily diet and exercise by themselves, there was no program providing immediate feedback or motivational messages to participants on whether their daily goal amount was achieved. Additionally, the interventions in reviewed studies were developed without a theoretical background or conceptual model. Therefore, we developed an innovative theory-based, two-way interactive, simple, and convenient intervention program that enables obese children to self-manage by providing information and immediate feedback or motivation related to obesity management using a mobile device.

\section{2) Step 2}

Construction of the mobile-based self-management health alarm program based on the information-motivation-behavioral skills model Based on the results of step 1, the authors developed the MSHA program based on the IMB model. The entire content of the developed intervention was provided only to study participants via the developed mobile accessible web-based platform and SNS text message. A summary of the MSHA program is presented in Table 1. The "Information: Facts" section of the program was provided to participants as mobile-accessible educational material. It covered characteristics of childhood obesity, obesity complications, self-management of obesity through diet (including a goal for daily calorie intake and an overview of the benefits of nutritionally balanced diet), and exercise (including a goal for daily steps

Table 1. Summary of the Mobile-based Self-management Health Alarm Program Based on the Information-Motivation-Behavioral Skills Model

\begin{tabular}{|c|c|}
\hline $\begin{array}{l}\text { IMB model } \\
\text { components }\end{array}$ & Program strategy \\
\hline Information & $\begin{array}{l}\text { Facts: Education provided by mobile-accessible material } \\
\cdot \text { Obesity in childhood } \\
\cdot \text { Complications of obesity } \\
\cdot \text { Health behavior for self-health management of obesity } \\
\text { - Diet: Provided individual goals for daily calorie intake and an overview of the benefits of a nutritionally } \\
\quad \text { balanced diet } \\
\text { - Exercise: Provided individual goals for daily steps and an overview of the benefits of exercise }\end{array}$ \\
\hline \multirow[t]{2}{*}{ Motivation } & $\begin{array}{l}\text { - Attitude: Self-monitoring and uploading to the developed platform } \\
\text { - Upload calculated calorie intake using the dietary application } \\
\text { - Upload measured number of steps using wearable device } \\
\text { - Support: Motivation provided via mobile text message as a health alarm } \\
\text { - Achievement status for calorie intake and steps } \\
\text { - Encouragement for self-health behavior in terms of calorie intake and steps }\end{array}$ \\
\hline & $\begin{array}{l}\text { - Attitude: Self-monitoring and uploading to the developed platform } \\
\text { - Upload the final daily calorie intake calculated using the dietary application } \\
\text { - Upload the final daily number of steps measured using wearable device } \\
\text { - Support: Motivation provided via mobile text message as a health alarm } \\
\text { - Final goal achievement status for calorie intake and steps } \\
\text { - Encouragement to continue engaging in self-health behavior the next day }\end{array}$ \\
\hline Behavioral skills & $\begin{array}{l}\text { Objective skills: Healthy diet and exercise skills provided by mobile-accessible material } \\
\text { - Nutritionally balanced menu planning skills } \\
\text { - Recommended walking speed and posture skills }\end{array}$ \\
\hline
\end{tabular}

IMB, information-motivation-behavioral skills. 
and an overview of the benefits of exercise) based on clinical practice guidelines or recommendations for childhood obesity interventions $[5,20]$. The recommended goal for daily calorie intake of each child was calculated using the estimated energy requirements (EER) formula, which considers the child's age, height, weight, and activity level [21]. The formula for children aged 9-18 years was as follows: EER for boys $=88.5$ $(61.9 \times$ age $)+$ physical activity $\times(26.7 \times$ weight $+903 \times$ height $)$ +25 ; and EER for girls=135.3 $-(30.8 \times$ age $)+$ physical activity $\times(10 \times$ weight $+934 \times$ height $)+25$. In the formula, a physical activity level of "1" was applied, corresponding to the value of sedentary physical activity, considering the low physical activity level of obese Korean children [5]. The daily step goal was set at 10,000 steps for all participants, based on literature recommendations, which is equivalent to 60 minutes of moderate to vigorous physical activity [22,23]. The "Motivation: Attitude" section of the program was indirectly induced by the children uploading their self-monitored calorie intake and total number of daily steps to the MSHA mobile-accessible platform. This section was intended to stimulate an attitude within the participants to maintain healthy behaviors through self-monitoring and self-reporting their diet and exercise. "Motivation: Support" was provided twice per day (8 PM and 10 PM) by study facilitators (research assistants), who sent simple motivational text messages to participants via SNS as health alarms. The messages were based on each child's daily goal achievement status. The first health alarm was intended to encourage the child's self-monitoring and their catching-up on daily diet and exercise goals, and the second health alarm was intended to promote self-reflection on that day's achievement of health behavior and to encourage the child to continue engaging in self-health behavior the next day. Before providing the simple motivational text messages as a health alarm, children were asked to monitor and upload their measured calorie intake using the free public-use diet-diary phone app (DASHIN) and the number of steps they had taken, as measured by a wearable pedometer (Xiaomi Mi band 3, Xiaomi Corporation, Beijing, China). The data were uploaded to the online MSHA platform using a mobile device. A sample of the second motivational mobile text messages as a health alarm is shown in Table 2. The "Behavioral skills: Objective skills" section of the program was provided as mobile-accessible educational material regarding a nutritionally balanced diet plan, including a well-balanced array of foods and healthy alternative foods (e.g., drinking water instead of soda or eating more vegetables instead of meats), and walking skills, including good posture, stride, and speed.

\section{3) Step 3}

Evaluation of content validity and implementation of the mobile-based self-management health alarm program The content of the developed program was evaluated using the content validity index (CVI) (Likert scale; 1=very inappropriate, $4=$ very appropriate) by experts (three nursing professors and one medical professor). The mean value of the CVI ranged from 3.00 to 3.75 . Hence, the authors decided to implement the developed intervention program as a mobile-based program. However, two of the evaluators thought it would be

Table 2. Samples of Motivational Mobile Text Messages before Sleep as Health Alarms

\begin{tabular}{|c|c|c|}
\hline Categories & Condition & Motivational mobile text message \\
\hline \multirow[t]{2}{*}{ Diet } & $\begin{array}{l}\leq \text { Goal for daily calorie intake } \\
\text { (goal met) }\end{array}$ & $\begin{array}{l}\text { You are so cool. } \\
\text { You did a great job with today's diet. } \\
\text { See you tomorrow with good results. } \\
\text { We are with you and support you. }\end{array}$ \\
\hline & $\begin{array}{l}\text { > Goal for daily calorie intake } \\
\text { (goal not met) }\end{array}$ & $\begin{array}{l}\text { You exceeded your calorie intake goal. } \\
\text { Did you find it difficult to adjust your diet? } \\
\text { Let's try to reduce your calorie intake a little more tomorrow. } \\
\text { I hope that you achieve your goal tomorrow. You can do it. }\end{array}$ \\
\hline \multirow[t]{2}{*}{ Exercise } & $\begin{array}{l}\geq \text { Goal for daily steps } \\
\text { (goal met) }\end{array}$ & $\begin{array}{l}\text { You have achieved your goal today. } \\
\text { You did well and we are proud of you. } \\
\text { Tomorrow you will be able to achieve your goal, again. } \\
\text { See you tomorrow with good results. } \\
\text { We are with you and support you. }\end{array}$ \\
\hline & $\begin{array}{l}<\text { Goal for daily steps } \\
\text { (goal not met) }\end{array}$ & $\begin{array}{l}\text { You didn't walk a lot today. } \\
\text { Did you find it difficult to adjust your exercise habits? } \\
\text { Let's try to increase your steps a little more tomorrow. } \\
10,000 \text { steps per day! You can do it. }\end{array}$ \\
\hline
\end{tabular}


more convenient for the children to change the fixed time for data monitoring and uploading from $8 \mathrm{PM}$ to "before dinner" and from 10 PM to "before sleep", considering possible variations in the children's daily situations. In response to these recommendations, the times for self-monitoring and uploading the data and providing the motivational text messages were modified to "before dinner" and "before sleeping" to allow flexibility. The confirmed intervention program was developed by a specialized information technology (IT) company as a mobile accessible web platform (http://mshealthyalarm.online/). The developed MSHA platform was constructed in a simple and convenient manner with (1) a page explaining how the program operated; (2) an educational page for providing the "Information" and "Motivation skills" sections of the program, dealing with topics such as obesity and its complications, checking one's daily diet and exercise recommendations, and diet and exercise management skills; (3) a data monitor and upload page for diet and exercise amounts; and (4) an inquiry page. The "Motivation" page was built to automatically send mobile text messages based on the uploaded diet and exercise data, with specific messages according to whether participants had or had not achieved their goals for the day, as shown in Table 2.

\section{Phase II: Feasibility Test for Metabolic Outcomes}

\section{1) General characteristics of study participants}

The study participants were four boys $(66.7 \%)$ and two girls $(33.3 \%)$. The mean age of the study participants was 11.2 years. The mean sitting hours per day was 11.83 hours, the mean number of days per week when participants exercised for over 60 minutes was 1.33, and two (33.3\%) perceived their health as "unhealthy", three (50.0\%) as "moderate", and one (16.7\%) as "healthy". Regarding the medical history of their parents, two had diabetes(33.3\%), one had hypertension (16.7\%), and four had hyperlipidemia(66.7\%) (Table 3).

\section{2) Mean number of goal-achievement days per week for diet and exercise}

The mean number of days that participants achieved their goals of diet and exercise in each week (0-7 days) during the intervention was 5.3-6.0 for diet and 5.0-5.3 for exercise. In the 4-week period, the mean value of goal-achieved days for diet was $5.7 \pm 0.30$, and this value increased from 5.3 at week 1 to 6.0 at week 4 . For exercise, the mean number of days where goals were achieved was $5.2 \pm 0.17$, and it fluctuated between 5.0 and 5.3 as time passed from the first to the fourth week.

\section{Effects of the Mobile-based Self-management Health Alarm Program on Metabolic Outcomes}

Among metabolic components, BMI $(Z=-1.99, p=.046)$, WC $(Z=-2.20, p=.028)$, and TG levels $(Z=-2.21, p=.027)$ significantly decreased; thus, metabolic outcomes significantly improved from before the intervention to after the intervention. The other variables showed no significant differences (Table 4).

\section{DISCUSSION}

This study was conducted to develop an MSHA program based on the IMB model and to test its feasibility. The authors carried out a pilot study using a one-group pretest-posttest design to evaluate feasibility and effectiveness before applying the developed intervention program to a large population of obese children; thus, there are currently limitations in generalizing the results of this study to the general population.

Table 3. General Characteristics of Study Participants $(N=6)$

\begin{tabular}{llcc}
\hline Variables & Categories & $\mathrm{n}(\%)$ or M \pm SD & Min-Max \\
\hline Age (year) & & $11.2 \pm 1.0$ & $10-12$ \\
Sex & Male & $4(66.7)$ \\
& Female & $2(33.3)$ \\
Sitting hours per day (hour) & & $11.83 \pm 2.48$ & $9-15$ \\
Numbers of days of exercising over & & $1.33 \pm 1.03$ \\
60 minutes per week (day) & & \\
Perceived health status & Unhealthy & $2(33.3)$ \\
& Moderate & $3(50.0)$ \\
& Healthy & $1(16.7)$ \\
Family medical history* & Diabetes mellitus (yes) & $2(33.3)$ \\
& Hypertension (yes) & $1(16.7)$ \\
\end{tabular}

*Duplicate answer allowed; Max, maximum; Min, minimum. 
Table 4. Effects of the Mobile-based Self-management Health Alarm Program on Metabolic Outcomes $(N=6)$

\begin{tabular}{|c|c|c|c|c|}
\hline \multirow{2}{*}{ Variables } & Pretest & Posttest & Z & \multirow{2}{*}{$p$} \\
\hline & $\mathrm{M} \pm \mathrm{SD}$ & $\mathrm{M} \pm \mathrm{SD}$ & (Post-Pre) & \\
\hline BMI $\left(\mathrm{kg} / \mathrm{m}^{2}\right)$ & $30.91 \pm 4.83$ & $30.49 \pm 4.84$ & -1.99 & .046 \\
\hline $\mathrm{WC}(\mathrm{cm})$ & $94.50 \pm 10.98$ & $89.17 \pm 9.67$ & -2.20 & .028 \\
\hline SBP (mmHg) & $117.33 \pm 7.26$ & $115.83 \pm 7.76$ & -0.94 & .345 \\
\hline DBP (mmHg) & $68.33 \pm 14.87$ & $71.17 \pm 11.97$ & -1.29 & .197 \\
\hline $\mathrm{TG}(\mathrm{mg} / \mathrm{dL})$ & $149.50 \pm 47.53$ & $118.33 \pm 50.35$ & -2.21 & .027 \\
\hline HDL-C (mg/dL) & $48.50 \pm 10.73$ & $48.33 \pm 11.47$ & -0.32 & .750 \\
\hline FPS (mg/dL) & $101.50 \pm 23.89$ & $95.50 \pm 3.56$ & -0.74 & .461 \\
\hline
\end{tabular}

BMI, body mass index; DBP, diastolic blood pressure; FPS, fasting plasma glucose; HDL-C, high-density lipoprotein-cholesterol; SBP, systolic blood pressure; TG, triglycerides; WC, waist circumference.

Nevertheless, this study has significance in that it presents the development of a mobile-based intervention that helps children perform diet and exercise self-management for obesity management by themselves, without help from their parents, under the coordination of health care providers.

Similar to this study, a study by Bartholmae [11] using the IMB model to improve the eating, exercising, and fruit and vegetable consumption behavior of American children demonstrated that the components of the IMB model effectively influenced the practice of the targeted health behaviors. In particular, the knowledge and motivation components of the IMB model were shown to be the most powerful factors in the model for changing children's health behavior [11]. In this study, the MSHA program to strengthen children's knowledge, motivation, and behavioral skills for obesity self-management may have resulted in successful goal achievement on more than 5 days per week and maintenance of their daily health behaviors. In particular, based on the IMB model's theoretical assumption that providing motivation is important for behavior change, the successful completion of the program by all participants without dropout indicates that intensively providing motivation in the form of health alarms was effective in changing these obese children's behavior.

Some previous studies have identified the effectiveness of mobile-based interventions for obese children [14,19]. The identified advantages of mobile-based interventions were that subjects could easily access the information provided by the healthcare provider anytime, anywhere, and without a wait time or cost, and they could immediately check whether they were achieving their targeted health behavior. In this study, the uploaded health educational materials, which included diet and exercise skills, on the MSHA platform could be browsed at any time using mobile devices. In addition, the platform allowed children themselves to easily check their goal achievement status. This process assigned responsibility to the children and motivated them to take care of their health on a regular basis. Additionally, mobile-based interventions provide several advantages compared to in-person healthcare providers for maximizing the effectiveness of interventions by monitoring patients' data and providing planned interventions anytime and anywhere [6,14]. This study had those advantages. It was also convenient for the researchers to evaluate the status of participants' daily goal achievements according to the uploaded data on the MSHA platform and to immediately provide constructed motivational text messages of what steps the child should take next for their self-management behaviors on that day or the following day. This ability to manage the health of many subjects with a small number of healthcare providers could also be considered a benefit of the program in terms of cost-effectiveness.

Moreover, a systematic review suggested that providing text messages is strongly effective in reducing the dropout rate of study participants [14]. Similar results were confirmed in this study; all study participants were fully engaged and successfully completed the 4-week program, and the mean number of goal achievement days per week was more than 5 days for both diet and exercise. In particular, before the intervention engagement, the mean number of days per week when the participants exercised for over 60 minutes was only 1.33. This finding may be interpreted as indicating that the study participants were motivated to maintain health behavior through the motivational text messages that were automatically provided twice per day. Moreover, mobile-based interventions can be an extremely effective method if a child does not want to receive treatment because of fear of treatment or social stigma [6]. With these advantages, the MSHA program may be a user-friendly and beneficial program for children who repeatedly fail to attend other intervention programs or who do not receive active treatment due to peer bullying or low self-esteem caused by obesity. 
Another notable benefit of the MSHA program was the improvement of metabolic outcomes. Among the measured metabolic components in this study, BMI, WC, and TG levels significantly decreased by the end of the intervention. According to a previous study [24], obese adolescents with a BMI more than the 95th percentile had 3.5-fold higher adult cardiovascular mortality than adolescents with a BMI in the 5-24th percentiles. That study verified the importance of childhood $\mathrm{BMI}$ as an important index for predicting cardiovascular disease in adulthood, and confirmed the urgent need for interventions to reduce BMI in obese children. From this perspective, the MSHA program, which led to a significant decrease in children's BMI, could be effective in reducing the prevalence of metabolic syndrome in adulthood by reducing BMI in childhood. Therefore, a future study with a larger sample is needed to evaluate the long-term effectiveness of the MSHA program. Moreover, in this study, the mean WC significantly decreased from $94.50 \mathrm{~cm}$ at the pretest to $89.17 \mathrm{~cm}$ at the posttest. WC is the best indirect predictor of visceral fat accumulation in children and is associated with increased insulin resistance [25-27]. According to a study by Morrison et al. [28], the risk of developing metabolic syndrome in girls aged $9-10$ years increased by $7.4 \%$ over a 10 -year period when their WC increased by $1 \mathrm{~cm}$ for every 2 years. Our findings showed that the MSHA program could be effective in reducing the prevalence of metabolic syndrome through a reduction in WC. Lastly, the mean TG level significantly decreased from $149.50 \mathrm{mg} / \mathrm{dL}$ to $118.33 \mathrm{mg} / \mathrm{dL}$ in the children in the MSHA program study. According to previous studies, the risk of metabolic syndrome increased by $1.3 \%$ when TG levels increased by $1 \mathrm{mg} / \mathrm{dL}$ in children [28], and the incidence of cardiovascular disease in adulthood increased 5 times when hypertriglyceridemia developed in childhood, as compared to in adulthood [29]. These results confirmed that the reduction in TG levels could contribute to reduction in the incidence of metabolic syndrome and cardiovascular disease in the future, especially when TG levels decrease following reduced intake of simple sugars and saturated or trans fats [29]. The decrease in TG levels in this study could have resulted from the MSHA program helping children monitor and maintain their daily calorie intake for 4 weeks with a low-sugar and low-fat diet. Therefore, the MSHA program was feasible and effective in engaging and maintaining obese children's self-management health behavior and improving metabolic outcomes.

However, this study has limitations. First, the small sample size limited our ability to examine potential covariates and generalizability of the findings to all children. Second, due to the lack of a control group and randomization as an experimental study, the results should be interpreted with caution, and causality could not be established. Future randomized controlled trials with larger samples and consideration of influencing factors are needed. Third, the data of daily exercise and diet levels were collected solely by the child's self-reports, so care must be taken about the objectivity of the collected data. Fourth, long-term effects could not be examined in this study. The effectiveness of the MSHA program was examined through the number of days per week when children achieved their goals for diet and exercise and improvements metabolic outcomes for only 4 weeks. The lasting effects of the MSHA program were not evaluated. Considering the findings of a previous study [13] that an online intervention for obesity prevention showed significant weight loss up to the first 6 months, but no significant difference was observed at follow-up after 2 years, further research is needed to evaluate possible long-term effects after the intervention. Lastly, the direct effect of each component of the IMB model on children's knowledge, motivation, and behavioral skills was not evaluated, but only assumed in light of the final outcomes in this study. Determining which component of IMB has the strongest influence on children's behavior could inform tailored interventions for this population with higher effectiveness. Therefore, a future study should consider evaluating the elements of the IMB model to improve the effectiveness of the developed interventional program.

The clinical significance of this study was that the MSHA program developed herein allows nursing health care providers to monitor and provide motivation for children's diet and exercise without situational restrictions, targeting lateschool-age obese children who are sufficiently familiar with the use of mobile devices to be called the "mobile native generation." Furthermore, the theoretical significance of this study lies in its application of the IMB model to the development of a nursing intervention program aimed at maintaining the health behavior of obese children.

\section{CONCLUSION}

To change and enhance obese children's diet and exercise self-management health behavior, the MSHA program was developed based on the IMB model and evaluated for its effectiveness and feasibility. The IMB model was well suited as a conceptual framework to develop a self-management interventional program for obese children. The MSHA program showed positive effectiveness for reducing metabolic syndrome risk and improving self-health management behavior in obese children by themselves. Based on the results of this pilot study, the MSHA program could be applied to a large population of late-school-age children. Furthermore, in view of the effectiveness of the program for improving metabolic outcomes, future studies that apply the MSHA program could 
be expected to prevent metabolic syndrome.

\section{Conflict of interest}

No existing or potential conflict of interest relevant to this article was reported.

\section{Data availability}

Please contact the corresponding author for data availability.

\section{REFERENCES}

1. World Health Organization. Commission on Ending Childhood Obesity: Taking action onchildhood obesity report [Internet]. Geneva: World Health Organization; 2018 September 25 [cited 2020 March 25]. Available from: https://www.who.int/end-childhood-obesity/en/

2. Lim J. 1 in 3 People is obese $\cdots$ OECD "Korean high obesity doubles by 2030". Newsis [Internet]. 2018 July 26 [cited 2020 March 25]; Local. Available from: https://www.newsis.com/view?id=NISX20180726_0000374805

3. Agirbasli M, Tanrikulu AM, Berenson GS. Metabolic syndrome: Bridging the gap from childhood to adulthood. Cardiovascular Therapeutics. 2016;34(1):30-36.

https://doi.org/10.1111/1755-5922.12165

4. The GBD 2015 Obesity Collaborators. Health effects of overweight and obesity in 195 countries over 25 years. New England Journal of Medicine. 2017;377(1):13-27.

https://doi.org/10.1056/NEJMoa1614362

5. Chung S. Therapeutic approaches to obesity and metabolic syndrome in children and adolescents. Journal of the Korean Medical Association. 2018;61(10):599-606.

https://doi.org/10.5124/jkma.2018.61.10.599

6. Sorgente A, Pietrabissa G, Manzoni GM, Re F, Simpson S, Perona S, et al. Web-based interventions for weight loss or weight loss maintenance in overweight and obese people: A systematic review of systematic reviews. Journal of Medical Internet Research. 2017;19 (6):e229. https://doi.org/10.2196/jmir.6972

7. Chang SJ, Choi SY, Kim SA, Song MS. Intervention strategies based on information-motivation-behavioral skills model for health behavior change: A systematic review. Asian Nursing Researh. 2014; 8(3):172-181. https://doi.org/10.1016/j.anr.2014.08.002

8. Fisher WA, Fisher JD, Harman J. The information-motivation -behavioral skills model: A general social psychological approach to understanding and promoting health behavior. In: Suls J, Wallston KA, editors. Social psychological foundations of healthand illness. Oxford: Blackwell Publishing Ltd.; 2003. p. 82-106. https://doi.org/10.1002/9780470753552.ch4

9. Zhu C, Cai Y, Ma J, Li N, Zhu J, He Y, et al. Predictors of intention to smoke among junior high school students in Shanghai, China: An empirical test of the information-motivation-behavioral skills (IMB) model. PLoS One. 2013;8(11):e80482.

https://doi.org/10.1371/journal.pone.0080482

10. Lee HY, Choi EK, Kim HJ, Kim HS, Kim HS. Factors affecting the self-management of adolescents with type 1 diabetes mellitus based on the information-motivation-behavioral skills model. Child Health Nursing Research. 2019;25(2):234-243.

https://doi.org/10.4094/chnr.2019.25.2.234

11. Bartholmae MM. The information-motivation-behavioral skills model: An examination of obesity prevention behavioral change in children who participated in the afterschool program Virginia Beach let's move [dissertation]. Norfolk, VA: Old Dominion University; 2016. p. 1-137. https://doi.org/10.25777/xv08-0b09

12. Jang BS, Kim DH. Health literacy and health behavior in late school-age children. Journal of Korean Academy of Community Health Nursing. 2015;26(3):199-208.

https://doi.org/10.12799/jkachn.2015.26.3.199

13. Park BK. Systematic review on the online adolescent obesity prevention program. Journal of Kyungpook Nursing Science. 2018;22 (1):41-50.

14. Lee J, Piao M, Byun A, Kim J. A systematic review and meta-analysis of intervention for pediatric obesity using mobile technology. Studies in Health Technology and Informatics. 2016;225:491-494. https://doi.org/10.3233/978-1-61499-658-3-491

15. Cho H, Yang JH, Lee J. A study on the wearable product design for promoting effects of children's excercise: Focused on movementmonitoring smart clothing design using textile sensor. Journal of the Korean Society of Design Culture. 2017;23(1):603-612.

https://doi.org/10.18208/ksdc.2017.23.1.603

16. Jeong Y. Intergenerational changes in media use and spread of smart phones. Korea Information Society Development Institute STAT Report. Jincheon: Korea Information Society Development Institute; 2015 April. Report No.: 15-01.

17. Kim MJ, Kim HS, Kim S, Kim DH. Development of an obesity management program for elementary school children: Based on Keller's Motivation Theory. Child Health Nursing Research. 2007; 13(3):257-264.

18. Kim JH, Yun S, Hwang SS, Shim JO, Chae HW, Lee YJ, et al. The 2017 Korean National Growth Charts for children and adolescents: Development, improvement, and prospects. Clinical and Experimental Pediatrics. 2018;61(5):135-149. https://doi.org/10.3345/kjp.2018.61.5.135

19. Smith JJ, Morgan PJ, Plotnikoff RC, Dally KA, Salmon J, Okely AD, et al. Smart-phone obesity prevention trial for adolescent boys in low-income communities: The ATLAS RCT. Pediatrics. 2014;134(3): e723-e731. https://doi.org/10.1542/peds.2014-1012

20. Anderson KL. A review of the prevention and medical management of childhood obesity. Child and Adolescent Psychiatric Clinics of North America. 2018;27(1):63-76. 
https://doi.org/10.1016/j.chc.2017.08.003

21. Nelms M. Nutrition therapy and pathophysiology. 3nd ed. Boston, MA: Cengage Learning; 2016.

22. Kim Y, Yang Y, Park H, Kim J. Development of physical activity guidelines and self-prescription guides for Korean. Research Report of Health Promotion Research Project. Seoul: Korea Health Promotion Institute (Seoul National University); 2012 November. Report No.: TRKO201500007063.

23. Tudor-Locke C, Craig CL, Beets MW, Belton S, Cardon GM, Duncan S, et al. How many steps/day are enough? for children and adolescents. International Journal of Behavioral Nutrition and Physical Activity. 2011;8:78. https://doi.org/10.1186/1479-5868-8-78

24. Twig G, Yaniv G, Levine H, Leiba A, Goldberger N, Derazne E, et al. Body-mass index in 2.3 million adolescents and cardiovascular death in adulthood. New England Journal of Medicine. 2016;374 (25):2430-2440. https://doi.org/10.1056/NEJMoa1503840

25. Lee J. Clinical predictive factors for metabolic syndrome in obese children and adolescents. Journal of Obesity and Metabolic Syndrome. 2016;25(2):50-55. https://doi.org/10.7570/kjo.2016.25.2.50

26. Brambilla P, Bedogni G, Moreno LA, Goran MI, Gutin B, Fox KR, et al. Crossvalidation of anthropometry against magnetic resonance imaging for the assessment of visceral and subcutaneous adipose tissue in children. International Journal of Obesity. 2006;30(1):2330. https://doi.org/10.1038/sj.ijo.0803163

27. Després JP, Lemieux I. Abdominal obesity and metabolic syndrome. Nature. 2006;444(7121):881-887.

https://doi.org/10.1038/nature05488

28. Morrison JA, Friedman LA, Harlan WR, Harlan LC, Barton BA, Schreiber GB, et al. Development of the metabolic syndrome in black and white adolescent girls: A longitudinal assessment. Pediatrics. 2005;116(5):1178-1182. https://doi.org/10.1542/peds.2004-2358

29. Morrison JA, Glueck CJ, Woo JG, Wang P. Risk factors for cardiovascular disease and type 2 diabetes retained from childhood to adulthood predict adult outcomes: The Princeton LRC followup study. International Journal of Pediatric Endocrinology. 2012;2012 (1):6. https://doi.org/10.1186/1687-9856-2012-6 